\title{
Rolling down to D-brane and tachyon matter
}

\author{
AKIRA ISHIDA* and SHOzO UEHARA ${ }^{\dagger}$ \\ Department of Physics, Nagoya University, \\ Chikusa-ku, Nagoya 464-8602, Japan
}

\begin{abstract}
We investigate the spatially inhomogeneous decay of an unstable D-brane and construct an asymptotic solution which describes a codimension one D-brane and the tachyon matter in boundary string field theory. In this solution, the tachyon matter exists around the lower-dimensional D-brane.
\end{abstract}

\section{Introduction}

Time dependent solutions of tachyon have been vigorously investigated recently. One of the purpose is to study the decay of an unstable D-brane. It was shown in [1, 2] that unstable D-branes become the pressure-less gas with non-zero energy density at late time, which is called tachyon matter, if the tachyon rolls down to the bottom of the potential homogeneously. While the time dependent solution is described by the boundary state, effective field theory approaches give similar results. The Born-Infeld type effective field theory [3]-[5] reproduces the known results of the rolling tachyon [6, 7]. In [8, 9], the spatially homogeneous decay was analyzed by using boundary string field theory (BSFT) [10]-[16]. They gave the asymptotic solution $\dot{T} \sim \pm 1$, which reproduces the property of the tachyon matter. Since the unstable $\mathrm{D} p$-brane can decay into $\mathrm{D}(p-1)$-brane, it is interesting to consider the spatially inhomogeneous tachyon which represents the time evolution of the kink solution. In this paper, we study the behavior of the spatially inhomogeneous decay of an unstable D-brane in BSFT and give an asymptotic solution. As we will see later, this solution asymptotically approaches $\dot{T} \sim \operatorname{sgn}\left(x^{0}\right)(\operatorname{sgn}(\cdot)$ : sign-function), and the lower-dimensional D-brane appears with the tachyon matter.

This paper is organized as follows. In section 2 we consider the inhomogeneous decay of an unstable D-brane and construct the asymptotic solution which describes both the lower-dimensional D-brane and the tachyon matter. Then we briefly discuss how the energy concentrates to make the lower-dimensional D-brane in section 3. The final section is devoted to conclusion and discussion.

*e-mail:ishida@eken.phys.nagoya-u.ac.jp

$\dagger$ †e-mail:uehara@eken.phys.nagoya-u.ac.jp 


\section{Inhomogeneous decay of the unstable D-brane}

In this section, we consider the late time behavior of the decaying D-brane into the lowerdimensional D-brane in BSFT. ${ }^{1}$ The action is given by ${ }^{2}[14$

$$
S=-T_{p} \int d^{p+1} x e^{-\frac{1}{4} T^{2}} \mathcal{F}\left(\eta^{\mu \nu} \partial_{\mu} T \partial_{\nu} T\right)
$$

where

$$
\mathcal{F}(x)=\frac{x 4^{x} \Gamma(x)^{2}}{2 \Gamma(2 x)},
$$

and $T_{p}$ is the tension of a non-BPS D-brane. The energy-momentum tensor obtained from this action is

$$
T_{\mu \nu}=T_{p} e^{-\frac{1}{4} T^{2}}\left[2 \partial_{\mu} T \partial_{\nu} T \mathcal{F}^{\prime}-\eta_{\mu \nu} \mathcal{F}\right] .
$$

Note that the energy-momentum conservation and the equation of motion are related as

$$
\begin{aligned}
\partial_{\mu} T^{\mu \nu} & =T_{p} \partial^{\nu} T\left\{2 \partial_{\mu}\left(e^{-\frac{1}{4} T^{2}} \partial^{\mu} T \mathcal{F}^{\prime}\right)+\frac{T}{2} e^{-\frac{1}{4} T^{2} \mathcal{F}}\right\} \\
& =-\partial^{\nu} T\left\{\partial_{\mu}\left(\frac{\partial \mathcal{L}}{\partial\left(\partial_{\mu} T\right)}\right)-\frac{\partial \mathcal{L}}{\partial T}\right\} .
\end{aligned}
$$

In order that the codimension one D-brane, ${ }^{3}$ i.e. kink solution, will be developed finally, we set the initial condition at $x^{0}=0$ as

$$
T>0 \quad\left(x^{1}>0\right), \quad T\left(x^{1}\right)=-T\left(-x^{1}\right), \quad \dot{T}=0,
$$

and we also impose that the initial value of the tachyon field is very small, i.e. $|T| \ll 1$ at $x^{0}=0$. Having imposed the initial conditions, we expect that $T \rightarrow \infty$ for $x^{1}>0$ and $T \rightarrow-\infty$ for $x^{1}<0$ at late time and the kink solution is dynamically generated. Due to the energy conservation, it is natural to expect that some non-zero energy density remains in the infinite region as well. ${ }^{4}$

Since we are interested in the inhomogeneity transverse to the codimension one D-brane, we put $\partial_{i} T=0$ for $i=2, \cdots, p$. Then the action becomes

$$
S=-T_{p} \int d^{p+1} x e^{-\frac{1}{4} T^{2}} \mathcal{F}(z),
$$

where

$$
z=-\dot{T}^{2}+T^{2} \quad\left(\dot{T} \equiv \partial_{0} T, T^{\prime} \equiv \partial_{1} T\right) .
$$

The hamiltonian density is

$$
\mathcal{H}=T^{00}=T_{p} e^{-\frac{1}{4} T^{2}}\left(\mathcal{F}(z)+2 \dot{T}^{2} \mathcal{F}^{\prime}(z)\right) .
$$

\footnotetext{
${ }^{1}$ Inhomogeneous rolling tachyon has been discussed also in [17]- 25].

${ }^{2}$ We set $\alpha^{\prime}=2$ for simplicity and the signature of the metric is taken to be $\{-,+,+, \cdots,+\}$. This action is exact as long as $\partial_{\mu} \partial_{\nu} T=0$ and higher derivative corrections are difficult to examine in BSFT. Since some reliable results have been obtained in [8, 9] and other papers, we neglect the higher derivative terms and proceed to investigate.

${ }^{3}$ We take the codimension one D-brane to be localized transverse to $x^{1}$-direction.

${ }^{4} \mathrm{~A}$ possibility that the energy dissipates to infinity is excluded as will be shown in section 3
} 
Let us investigate the equation of motion,

$$
e^{-\frac{1}{4} T^{2}}\left(\partial^{\mu} T \partial_{\mu} z \mathcal{F}^{\prime \prime}(z)+\partial^{\mu} \partial_{\mu} T \mathcal{F}^{\prime}(z)-\frac{1}{2} T z \mathcal{F}^{\prime}(z)+\frac{1}{4} T \mathcal{F}(z)\right)=0 .
$$

We assume that $z$ does not cross the singularity at $z=-1$ and restrict ourselves to $z>-1$, where $\mathcal{F}$ is analytic.

First, let us consider that $z$ goes to some finite value other than -1 , i.e. does not hit a singularity as $x^{0} \rightarrow \infty$. Since we treat the case where $|T| \rightarrow \infty$ as $x^{0} \rightarrow \infty$, the leading contribution to the equation of motion (9]) leads to (cf. ref.[26])

$$
D(z)=\mathcal{F}(z)-2 z \mathcal{F}^{\prime}(z)=0
$$

This equation has no solutions for finite values of $z$ because $D(z)$ is positive in $-1<z \leq 0$ [8] and it is also positive for $z>0$ since it can be written in that region as

$$
D(z)=2 \mathcal{F}(z) \int_{0}^{1} \frac{t^{2 z}}{(1+t)^{2}} d t \quad(z>0)
$$

In the case of $z \rightarrow \infty$, we obtain the same equation as eq.(10) because the asymptotic forms of $\mathcal{F}(y)$ and $\mathcal{F}^{\prime}(y)$ for the argument $y$ being large are given by

$$
\mathcal{F}(y)=\sqrt{\pi y}+\mathcal{O}\left(y^{-1 / 2}\right), \quad \mathcal{F}^{\prime}(y)=\frac{1}{2} \sqrt{\frac{\pi}{y}}+\mathcal{O}\left(y^{-3 / 2}\right) .
$$

And we see that $z \rightarrow \infty$ is a solution for eq.(10).

Next, we proceed to the case that $z \rightarrow-1$ as $x^{0} \rightarrow \infty$. We may write $|\dot{T}|=1-\mathcal{O}(\delta)$, $|\delta| \ll 1$ at late time, and assume $T^{\prime}=\mathcal{O}(\delta)$. Then we see that $z+1=\mathcal{O}(\delta)$ and $\dot{z}=\mathcal{O}(\delta)$. Since $\mathcal{F}(y), \mathcal{F}^{\prime}(y)$ and $\mathcal{F}^{\prime \prime}(y)$ behave near $y=-1$ as

$$
\mathcal{F}(y) \sim \frac{-1}{2(y+1)}, \quad \mathcal{F}^{\prime}(y) \sim \frac{1}{2(y+1)^{2}}, \quad \mathcal{F}^{\prime \prime}(y) \sim \frac{-1}{(y+1)^{3}},
$$

the leading contribution of eq.(9) in this case becomes

$$
2 \dot{T} \dot{z} \mathcal{F}^{\prime \prime}(z)-T z \mathcal{F}^{\prime}(z)=0,
$$

which leads to, due to eq.(13),

$$
\frac{\dot{z}}{z+1}=\frac{T z}{4 \dot{T}}=\frac{T\left(-\dot{T}^{2}+T^{2}\right)}{4 \dot{T}} \simeq-\frac{T \dot{T}}{4} .
$$

Solving this equation, we obtain the leading term of $z+1$ as

$$
z+1=g\left(x^{1}\right) e^{-\frac{1}{8} T^{2}},
$$

where $g\left(x^{1}\right)$ is a function of $x^{1} \cdot{ }^{5}$ Note that eqs.(16) and (8) implies finite energy density at late time. We stress here that the equation of motion requires that $z$ goes to either -1 or

\footnotetext{
${ }^{5}$ The $g\left(x^{1}\right)=$ const case corresponds to the $\epsilon\left(x^{0}\right)$ in $[8]$.
} 
infinity as $x^{0} \rightarrow \infty$, which generally holds when we consider the rolling tachyon. The static solution which represents the codimension one D-brane is $T=u x^{1}(u \rightarrow \infty)$ [14 and the spatially homogeneous solution for the rolling tachyon is $|\dot{T}| \rightarrow 1$ as $x^{0} \rightarrow \infty$ [2]. Thus, considering the above analysis of the equation of motion, we require that the asymptotic solution satisfies

$$
\begin{array}{ll}
T=0, \quad T^{\prime} \rightarrow \infty & \left(x^{1}=0\right), \\
\dot{T} \rightarrow \operatorname{sgn}\left(x^{1}\right), \quad T^{\prime} \rightarrow 0 & \left(x^{1} \neq 0\right) .
\end{array}
$$

Let us construct the solution describing the decay of an unstable D-brane into the lower-dimensional one. We expect the asymptotic solution for $T$ as

$$
T=\sigma(x)\left(x^{0}-\epsilon(x)\right) \quad\left(x=\left(x^{0}, x^{1}\right)\right),
$$

where $\epsilon(x)$ is a small perturbation and $\sigma(x)$ is a function which satisfies

$$
\sigma(x) \sim \operatorname{sgn}\left(x^{1}\right) \quad\left(x^{0} \rightarrow \infty\right) .
$$

First, we consider the asymptotic behavior of the tachyon for $x^{1} \neq 0$. In this region, $|T| \sim x^{0}$ and hence $z \rightarrow-1$. We have

$$
\begin{aligned}
z+1 & =1-\left\{\sigma(1-\dot{\epsilon})+\dot{\sigma}\left(x^{0}-\epsilon\right)\right\}^{2}+\left\{\sigma^{\prime}\left(x^{0}-\epsilon\right)-\sigma \epsilon^{\prime}\right\}^{2} \\
& \simeq 1-\sigma^{2}+2 \dot{\epsilon}-2\left(\operatorname{sgn}\left(x^{1}\right) x^{0}\right) \dot{\sigma} \\
& \simeq 2(1-|\sigma|)+2 \dot{\epsilon}-2\left(\operatorname{sgn}\left(x^{1}\right) x^{0}\right) \dot{\sigma}
\end{aligned}
$$

where use has been made of eq.(19) and we have assumed that $\dot{\sigma}$ and $\sigma^{\prime}$ are small. We also require, at late time,

$$
|\dot{\epsilon}| \gg|(1-|\sigma|)| \quad \text { and } \quad|\dot{\epsilon}| \gg x^{0}|\dot{\sigma}|
$$

Thus, in order to satisfy eq.(16), or obtain the finite energy solution, $\epsilon(x)$ is given by

$$
\epsilon(x)=f\left(x^{1}\right) \int_{\infty}^{x^{0}} d y e^{-\frac{1}{8} y^{2}}=-\sqrt{2 \pi} f\left(x^{1}\right) \operatorname{erfc}\left(x^{0} / \sqrt{8}\right),
$$

where $f\left(x^{1}\right)$ is a function of $x^{1}$ and we assume that it does not vary so much. Then we have

$$
e^{-\frac{1}{4} T^{2}} \mathcal{F}(z) \rightarrow 0, \quad e^{-\frac{1}{4} T^{2}} \mathcal{F}^{\prime}(z) \rightarrow \frac{1}{8 f^{2}}
$$

We shall proceed to $\sigma(x)$. From eqs.(16), (19) and (21), the difference, $\sigma(x)-\operatorname{sgn}\left(x^{1}\right)$, should damp more rapidly than $\exp \left(-\left(x^{0}\right)^{2} / 8\right)$ at late time. Thus we find that $\sigma(x)$ can be given by

$$
\sigma(x)=\frac{2}{\sqrt{\pi}} \int_{0}^{c\left(x^{0}\right)^{1+\alpha} x^{1}} d y e^{-y^{2}}=\operatorname{erf}\left(c\left(x^{0}\right)^{1+\alpha} x^{1}\right), \quad(\alpha>0)
$$

where $\alpha$ is a positive constant and $c$ is a constant, which can be taken positive without lost of generality. Although we write the asymptotic form of $T$ as eq.(18), note that $T$ is correct up to $o\left(e^{-\left(x^{0}\right)^{2} / 8}\right)$ except the infinitesimally small region around $x^{1}=0$. 
Next, we consider the behavior of the tachyon at the remaining point, i.e. $x^{1}=0$ as $x^{0} \rightarrow \infty$ with the above $\epsilon(x)(22)$ and $\sigma(x)$ (24). At this point we first notice $T=0$ due to eq.(24). Also we find that $T^{\prime} \simeq 2 c\left(x^{0}\right)^{2+\alpha} / \sqrt{\pi} \rightarrow \infty$ while $\dot{T}=0$, and hence $z \rightarrow \infty$. The asymptotic forms of $\mathcal{F}(y)$ and $\mathcal{F}^{\prime}(y)$ for the argument $y$ being large are given in eq. (12). Hence at exactly $x^{1}=0$, we find the following behavior for $x^{0} \rightarrow \infty$,

$$
e^{-\frac{1}{4} T^{2}} \mathcal{F}(z) \rightarrow \infty, \quad e^{-\frac{1}{4} T^{2}} \mathcal{F}^{\prime}(z) \rightarrow 0
$$

Let us calculate more carefully. In the infinitesimally small region around $x^{1}=0$ for $x^{0} \gg 1, T$ in eq.(18) and its derivatives are evaluated as

$$
T \simeq \frac{2}{\sqrt{\pi}} c\left(x^{0}\right)^{2+\alpha} x^{1}, \quad \dot{T} \simeq \frac{2(2+\alpha)}{\sqrt{\pi}} c\left(x^{0}\right)^{1+\alpha} x^{1}, \quad T^{\prime} \simeq \frac{2}{\sqrt{\pi}} c\left(x^{0}\right)^{2+\alpha} .
$$

Of course, this satisfies leading contribution of eq.(10), ${ }^{6}$ and eq.(12) leads to

$$
\begin{aligned}
e^{-\frac{1}{4} T^{2}} \mathcal{F}(z) & \simeq 2 c\left(x^{0}\right)^{2+\alpha} e^{-\frac{c^{2}}{\pi}\left(x^{0}\right)^{2(2+\alpha)}\left(x^{1}\right)^{2}} \rightarrow 2 \pi \delta\left(x^{1}\right), \\
e^{-\frac{1}{4} T^{2}} \dot{T}^{2} \mathcal{F}^{\prime}(z) & \simeq(2+\alpha)^{2} c\left(x^{0}\right)^{\alpha}\left(x^{1}\right)^{2} e^{-\frac{c^{2}}{\pi}\left(x^{0}\right)^{2(2+\alpha)}\left(x^{1}\right)^{2}} \rightarrow 0 .
\end{aligned}
$$

From eqs.(23) and (27), we find that the hamiltonian density asymptotically becomes

$$
\mathcal{H} \rightarrow 2 \pi T_{p} \delta\left(x^{1}\right)+\frac{T_{p}}{4\left\{f\left(x^{1}\right)\right\}^{2}} s\left(x^{1}\right)
$$

where

$$
s(y)=\left\{\begin{array}{ll}
1 & (y \neq 0) \\
0 & (y=0)
\end{array} .\right.
$$

This shows the correct tension $2 \pi T_{p}\left(=T_{p-1}\right)$ of the $\mathrm{D}(p-1)$-brane at $x^{1}=0$. The non-zero energy density remains and the pressure for $x^{0} \rightarrow \infty$ is given by plugging eqs.(17) and (23) into

$$
p=T^{11}=T_{p} e^{-\frac{1}{4} T^{2}}\left\{2\left(T^{\prime}\right)^{2} \mathcal{F}^{\prime}-\mathcal{F}\right\} .
$$

It is easy to check that the pressure falls off exponentially at late time. Thus, this non-zero energy density turns out to be the tachyon matter.

\section{Flow of energy}

Having found the asymptotic solution, we consider the momentum density, or the flow of energy in this section. Eq.(3) leads to

$$
T^{01}=-2 T_{p} e^{-\frac{1}{4} T^{2}} \dot{T} T^{\prime} \mathcal{F}^{\prime}(z) .
$$

We see that $T^{01}=0$ at $x^{1}=0$. From eqs.(18), (22) and (24), $\dot{T}$ and $T^{\prime}$ at late time except for $x^{1}=0$ are given by

$$
\begin{aligned}
\dot{T} & \simeq \operatorname{sgn}\left(x^{1}\right), \\
T^{\prime} & \simeq \sqrt{2 \pi} \operatorname{sgn}\left(x^{1}\right) f^{\prime}\left(x^{1}\right) \operatorname{erfc}\left(x^{0} / \sqrt{8}\right) .
\end{aligned}
$$

\footnotetext{
${ }^{6}$ The leading term of the equation of motion is also satisfied at $x^{1}=0$.
} 
The remaining factor of $e^{-T^{2} / 4} \mathcal{F}^{\prime}$ at late time is given in the previous section, so that it is easy to see that the energy ceases to flow at $x^{0} \rightarrow \infty$, which is, of course, consistent with the energy-momentum conservation. Note that if $f^{\prime}\left(x^{1}\right)=0$, or $f$ is constant, we cannot obtain explicit form of $T^{\prime}$ since eqs. (18) and (22) is correct up to $o\left(e^{-\left(x^{0}\right)^{2} / 8}\right)$. However, we see that $T^{\prime}$ is smaller than $\epsilon(x)$, which does not alter the above result at $x^{0} \rightarrow \infty$.

Although the flow of the energy at early time is not given, since we consider the time evolution of the kink, it is plausible that $\dot{T}>0\left(x^{1}>0\right), \dot{T}<0\left(x^{1}<0\right)$ and $T^{\prime}>0$ in some region around $x^{1}=0$ and hence the energy flows toward $x^{1}=0$ in some region around there after some finite time, which means that the energy does not dissipate to infinity. We note that the energy conservation implies $f\left(x^{1}\right) \sim 1 / 2$ if $f^{\prime}\left(x^{1}\right)$ is small.

\section{Conclusion and discussion}

We have investigated the spatially inhomogeneous decay of an unstable D-brane in boundary string field theory. We have considered the equation of motion and shown that $z$ must become either -1 or infinity as $x^{0} \rightarrow \infty$. We have also given the time dependent kink solution of tachyon eq.(18) with eqs.(22), (24) at late time. We found that the final state is the codimension one D-brane and the tachyon matter, the pressure-less gas with nonzero energy density. Furthermore, the energy (density) where the resultant codimension D-brane exists is $2 \pi T_{p}$ which exactly corresponds to the tension of the codimension one D-brane, $T_{p-1}=2 \pi T_{p}$. Recently the inhomogeneous rolling tachyon of a different profile has been analyzed in CFT approach [24. It is interesting to study the relation to our analysis.

Finally we comment on the excitation of gauge fields. In [27, gauge fields in the rolling tachyon was studied. We can perform a similar analysis with the rolling tachyon studied here and we find that the excitations of the gauge field can exist only on the lowerdimensional D-brane, while there are no excitations on the remaining tachyon matter.

Acknowledgments: The work of SU is supported in part by the Grant-in-Aid for Scientific Research No.13135212.

\section{References}

[1] A. Sen, "Rolling tachyon," JHEP 0204, 048 (2002) arXiv:hep-th/0203211.

[2] A. Sen, "Tachyon matter," JHEP 0207, 065 (2002) arXiv:hep-th/0203265.

[3] M. R. Garousi, "Tachyon couplings on non-BPS D-branes and Dirac-Born-Infeld action," Nucl. Phys. B 584, 284 (2000) arXiv:hep-th/0003122.

[4] E. A. Bergshoeff, M. de Roo, T. C. de Wit, E. Eyras and S. Panda, "T-duality and actions for non-BPS D-branes," JHEP 0005, 009 (2000) arXiv:hep-th/0003221.

[5] J. Kluson, "Proposal for non-BPS D-brane action," Phys. Rev. D 62, 126003 (2000) arXiv:hep-th/0004106. 
[6] A. Sen, "Field theory of tachyon matter," Mod. Phys. Lett. A 17, 1797 (2002) arXiv:hep-th/0204143.

[7] A. Sen, "Time and tachyon," arXiv:hep-th/0209122.

[8] S. Sugimoto and S. Terashima, "Tachyon matter in boundary string field theory," JHEP 0207, 025 (2002) arXiv:hep-th/0205085.

[9] J. A. Minahan, "Rolling the tachyon in super BSFT," JHEP 0207, 030 (2002) arXiv:hep-th/0205098.

[10] E. Witten, "On background independent open string field theory," Phys. Rev. D 46 , 5467 (1992) arXiv:hep-th/9208027.

[11] E. Witten, "Some computations in background independent off-shell string theory," Phys. Rev. D 47, 3405 (1993) arXiv:hep-th/9210065.

[12] A. A. Gerasimov and S. L. Shatashvili, "On exact tachyon potential in open string field theory," JHEP 0010, 034 (2000) arXiv:hep-th/0009103.

[13] D. Kutasov, M. Marino and G. W. Moore, "Some exact results on tachyon condensation in string field theory," JHEP 0010, 045 (2000) arXiv:hep-th/0009148.

[14] D. Kutasov, M. Marino and G. W. Moore, "Remarks on tachyon condensation in superstring field theory," arXiv:hep-th/0010108.

[15] M. Marino, "On the BV formulation of boundary superstring field theory," JHEP 0106, 059 (2001) arXiv:hep-th/0103089.

[16] V. Niarchos and N. Prezas, "Boundary superstring field theory," Nucl. Phys. B 619, 51 (2001) arXiv:hep-th/0103102.

[17] A. Sen, "Time evolution in open string theory," JHEP 0210, 003 (2002) arXiv:hep-th/0207105.

[18] N. Moeller and B. Zwiebach, "Dynamics with infinitely many time derivatives and rolling tachyons," JHEP 0210, 034 (2002) arXiv:hep-th/0207107.

[19] J. M. Cline, H. Firouzjahi and P. Martineau, "Reheating from tachyon condensation," JHEP 0211, 041 (2002) arXiv:hep-th/0207156.

[20] G. N. Felder, L. Kofman and A. Starobinsky, "Caustics in tachyon matter and other Born-Infeld scalars," JHEP 0209, 026 (2002) arXiv:hep-th/0208019.

[21] S. Mukohyama, "Inhomogeneous tachyon decay, light-cone structure and Dbrane network problem in tachyon cosmology," Phys. Rev. D 66, 123512 (2002) arXiv:hep-th/0208094.

[22] K. Hashimoto, P. M. Ho and J. E. Wang, "S-brane actions," arXiv:hep-th/0211090. 
[23] M. Berkooz, B. Craps, D. Kutasov and G. Rajesh, "Comments on cosmological singularities in string theory," arXiv:hep-th/0212215.

[24] F. Larsen, A. Naqvi and S. Terashima, "Rolling tachyons and decaying branes," JHEP 0302, 039 (2003) arXiv:hep-th/0212248.

[25] J. M. Cline and H. Firouzjahi, "Real-time D-brane condensation," arXiv:hep-th/0301101.

[26] K. Hashimoto and S. Hirano, "Metamorphosis of tachyon profile in unstable D9branes," Phys. Rev. D 65, 026006 (2002) arXiv:hep-th/0102174.

[27] A. Ishida and S. Uehara, "Gauge fields on tachyon matter," Phys. Lett. B 544, 353 (2002) arXiv:hep-th/0206102. 